https://doi.org/10.7559/gestaoedesenvolvimento.2019.378

Data de receção: 12/04/2019 Data de aceitação: 09/07/2019

\title{
INOVAÇÃO E OUTSOURCING: O CASO DA VOLKSWAGEN DO BRASIL
}

\section{INNOVATION AND OUTSOURCING: THE CASE OF VOLKSWAGEN IN BRAZIL}

\begin{abstract}
António José Oliveira ${ }^{1}$ orcid.org/0000-0002-8609-636X
Paulo Castro Ribeiro ${ }^{2}$ orcid.org/0000-0001-5662-6318
\end{abstract}

\begin{abstract}
Resumo: Atualmente as tecnologias de informação sustentam operações empresariais, unem elos distantes de cadeias de fornecimento e ainda, empresas a clientes. Neste artigo analisamos as motivações que levam uma empresa a promover outsourcing de atividades, ligadas aos seus processos de engenharia, tendo como foco principal o desenvolvimento de novos produtos, sem descurar os riscos associados ao outsourcing. O Business Process Outsourcing é uma decisão estratégica, que apresenta resultados a médio/longo prazo. $O$ trabalho empírico de suporte a este artigo intitula-se "Outsourcing nas atividades de desenvolvimento de novos produtos, o caso da Volkswagen do Brasil", elaborado por Pereira, Scur \& Mello. O estudo é qualitativo, pois foi realizado por amostragem, através de entrevistas presenciais, gravadas com autorização dos entrevistados. Este estudo contribui para o desenvolvimento da atividade empresarial. Identifica as razões pelas quais uma empresa do setor automóvel subcontrata "fornecedores" para realizar as suas atividades e desenvolver novos produtos.

Conclui-se que a opção pelo outsourcing está relacionada com a falta de um determinado recurso ou capacidade para realizar a
\end{abstract}

${ }^{1}$ Mestrando em Gestão, especialização em Gestão de Negócios da Universidade Católica Portuguesa, Viseu. E-mail: aj.oliveira@outlook.pt

2 Professor Auxiliar da Universidade Católica Portuguesa, Viseu. E-mail: pribeiro@viseu.ucp.pt 
atividade. Foram apontados como riscos do outsourcing, a fuga de conhecimento, a perda de capacidade crítica e consequentemente, a dependência em relação ao fornecedor e ainda, a capacidade da empresa em adquirir novas tecnologias.

Palavras-chave: Outsourcing, Inovação, Volkswagen.

Abstract: Today, information technology supports business operations, supply chain and business improvements for customers. This article analyzes the motivation that should be directed to the company to outsource activities, related to its engineering processes, concerned with the development of new products, taking into account the risks associated with outsourcing. Business Process Outsourcing is a strategic decision that presents results in the medium / long term. The empirical work that supports this work is titled "Outsourcing in the activities of development of new products, the case of Volkswagen do Brasil", elaborated by Pereira, Scur \& Mello. This study is qualitative and was performed by sampling, through face-to-face interviews, recorded with the consent of the interviewees. Its main objective was to contribute to the development of business activity. The study identifies the reasons why an automotive company subcontracts "suppliers" to carry out its activities and develop new products.

The conclusion of outsourcing is related to the lack of a resource for the ability to carry out an activity. They pointed out as risks of outsourcing, the leakage of knowledge, the loss of critical capacity and, consequently, the dependence on the supplier and finally the company's ability to acquire new technologies.

Keywords: Outsourcing, Innovation, Volkswagen.

\section{INTRODUÇÃO}

Os avanços das Tecnologias de Informação e Comunicação (TIC) levaram as empresas a poderem realizar de forma mais eficiente muitos processos, tais como: engenharia, desenvolvimento de TI, gestão de dados, faturação e atendimento ao cliente (Di Gregorio et al., 2009). As 
tecnologias de informação são cada vez mais um recurso estratégico para obter vantagem competitiva. São encaradas como um recurso essencial para o sucesso (Carr, 2003). A expansão das TI levou ao aparecimento de diversos tipos de estratégias, entre elas, o outsourcing. Optar pelo uso de ferramentas, como o outsourcing, implica que, tal decisão seja tomada pelos administradores/gestores, visto que leva a uma alteração dos limites da empresa, como entidade legal (Pereira et al., 2017).

Neste trabalho são analisadas as motivações que levam uma empresa a promover outsourcing de atividades, com implicação nos seus processos de engenharia, essencialmente ligadas ao desenvolvimento de novos produtos, considerando os riscos associados ao outsourcing.

Foi possível concluir, que a escolha pelo outsourcing está relacionada com a carência de um certo recurso ou capacidade de realização de uma determinada atividade. Foram, ainda, identificados os riscos do outsourcing.

Este artigo teve como base de trabalho, o estudo elaborado por Pereira, Scur \& Mello, intitulado "Outsourcing nas atividades de desenvolvimento de novos produtos, o caso da Volkswagen do Brasil", em 2017.

Quanto à estrutura do artigo, este está estruturado da seguinte forma: enquadramento teórico, estudo empírico, discussão de resultados, conclusões e referências bibliográficas.

\section{ENQUADRAMENTO TEÓRICO}

As organizações bem-sucedidas, independentemente da sua motivação pessoal, (dinheiro, poder, fama, reconhecimento) tentam criar novos valores, não se contentando somente, em melhorar ou modificar o que já existia. (Walter, Leite \& Craveiro, 2015).

\subsection{Inovação}

O conceito de Inovação é ainda um conceito em desenvolvimento. A sua natureza e contexto têm vindo a sofrer alterações ao longo do tempo, sofrendo a influência do país ou região em que se insere. Os primeiros dados de investigação deste fenómeno aconteceram, entre as décadas de 80 e 90. O progressivo desenvolvimento dessa investigação levou à criação do Manual de Oslo (Acosta, Acosta \& Espinoza, 2016). 
No conceito empresarial, a natureza das atividades de inovação é muito variável. Muitas empresas desenvolvem ou introduzem um novo produto, enquanto que outras realizam melhorias contínuas nos seus produtos, processos e operações (Oliveira et al., 2017).

Qualquer empresa com tendência inovadora, deve apoiar novas ideias, promover processos criativos destinados a desenvolver novos produtos e serviços. Deve ainda, tomar iniciativa de antecipar e procurar novas oportunidades de negócios e participar em mercados emergentes (Arenhardt, Simonetto \& Rodrigues, 2018).

A inovação empresarial é a criação de valor para os clientes e para a empresa, mudando de forma criativa, uma ou mais dimensões do sistema empresarial. A inovação está ligada à criação de novos valores e é multiforme. Pode ter lugar em qualquer dimensão de um negócio, é sistémica, logo, requer consideração cuidada de todas as vertentes de um negócio (Oliveira et al., 2017).

A inovação é um fator essencial na melhoria do desempenho do cliente. É encarada como uma recurso crucial para o sucesso. A sua utilização permite ganhar vantagem competitiva sobre os concorrentes. Esta só acontecerá, se clientes e fornecedores implementarem um processo mais amplo. Este processo deve combinar a aculturação entre diferentes organizações, um método que faça a gestão de ideias, financiamento adequado e um sistema para criação de mudanças. A maioria das ideias de inovação foi desenvolvida conjuntamente com clientes e fornecedores. Esta afirmação teve a concordância de prestadores de serviços, clientes e consultores ("Outsourcing Business Processes for Innovation.," 2013).

Os líderes impulsionam todos os níveis da organização a refletirem e repensarem os pressupostos, e a identificarem inovações, que melhorem o desempenho dos clientes. Estes não podem ser recetores passivos de inovação, mas sim, impulsionadores de mudança provocada pela inovação (Mcivor, 2011).

A realização de inovações dinâmicas pressupõem um forte compromisso com a organização, de modo a que indivíduos, equipas e unidades organizacionais transitem do estado atual para o futuro desejável. Uma liderança forte tem de integrar líderes fortes, que demonstrem os seguintes comportamentos: foco no futuro, foco nos 
resultados do cliente, espírito de união, transparência, orientação para a resolução de problemas e orientação para ação (Mcivor, 2011).

Fujimoto e Takeishi (2001) citado por Bueno \& Balestrin (2012) revelam que as empresas do ramo automóvel estabeleceram com os seus fornecedores parcerias, para o desenvolvimento de tecnologias, iniciando a abertura do processo de inovação.

Esta perspetiva interativa de colaboração entre a empresa e os seus colaboradores, já foi referenciada no trabalhos de Dyer e Nobeoka, em 2000 na apresentação do sistema "Toyota" (Bueno \& Balestrin, 2012).

\subsection{Outsourcing}

A necessidade de uma maior eficiência e redução de custos obrigou muitas empresas a inovar, especializando-se num número limitado de áreas-chave (Mcivor, 2011). Esta necessidade levou a que muitas empresas recorressem a vários tipos de estratégias de gestão, sendo uma delas, a subcontratação de atividades, também conhecida como outsourcing (Pereira et al., 2017).

O outsourcing pode ser definido como uma decisão estratégica de obtenção de recursos externos (produtos ou serviços) para substituir atividades tradicionalmente manipuladas por colaboradores internos e recursos (Aamer, 2018).

O Business Process Outsourcing (BPO) é uma decisão estratégica, que apresenta resultados a médio/longo prazo.

Segundo Maia (2003) citado por Pedriali (2004), o BPO consiste em transferir para um fornecedor externo a gestão operacional de um negócio, tornando acessível a infraestrutura de hardware, softwares aplicativos, etc (Pedriali, 2004).

Presentemente, o outsourcing de TI é tido como parte de uma transformação estratégica da função de TI, em que novas responsabilidades são transferidas para o prestador de serviços, fornecedor (Silva, 2009).

O sucesso do BPO advém da escolha de um bom parceiro de negócio, da definição dos limites de outsourcing necessárias à organização e do ajustamento dos processos, antes de considerar uma iniciativa de outsourcing (Pedriali, 2004). 
Tradicionalmente, a transferência de atividades de TI estava associada à redução de custos ou à resolução imediata de um problema (Silva, 2009).

Os mercados de fornecedores podem ser definidos como mercados de uma indústria estabelecida, onde existe maior concorrência entre vários fornecedores com processos de fabricação estabelecidos e um maior conhecimento e experiência no planeamento e controlo de operações. Este tipo de mercado comporta menos risco para as organização que utilizam atividades de outsourcing, relativamente aos mercados de fornecedores não desenvolvidos. Isto deve-se à maior capacidade dos níveis de controlo operacional dos fornecedores, traduzindo-se em maior confiança (Aamer, 2018).

Assim, em mercados de fornecedores desenvolvidos, a avaliação e seleção destes, não é tão rigorosa, relativamente aos mercados de fornecedores não desenvolvidos. Isto acontece, porque as organização que utilizam atividades de outsourcing têm dados históricos sobre o desempenho potencial do fornecedor, uma vez que estão integrados num mercado competitivo. Porém, em mercados de fornecedores não desenvolvidos, é preciso uma avaliação e seleção mais rigorosas dos fornecedores (Aamer, 2018).

Neste contexto, atividades em que uma organização tem uma capacidade superior, devem ser realizadas internamente, enquanto as atividades com uma capacidade inferior são candidatas a outsourcing (Mcivor, 2011).

Uma das desvantagens do outsourcing, é que muitas empresas cedem parte das atividades principais, a outros. Segundo Weibendaun (2005), tal não deverá acontecer, pois nenhuma empresa deverá terceirizar a gestão e a responsabilidade das suas atividades. Outras desvantagens apresentadas por este autor são: as empresas que terceirizam as suas atividades apenas porque os outros o fazem, podem ser surpreendidas por custos inesperados e diversas complicações, levando ao término de alguns dos acordos realizados, e ainda, a perda do emprego por parte dos funcionários, ou a sua deslocação para outras atividades da empresa, às quais não se sentem confortáveis.

Já, segundo Mcivor (2011), a ampliação das suas atividades em diversas áreas, prejudicará a sua competência essencial, bem como, os 
custos da realização devido à falta de experiência e especialização nestas áreas.

A vantagem principal do outsourcing é que, terceirizar pode ajudar a empresa a obter vantagem competitiva no mercado global. Assim, pode desenvolver as relações internacionais, reduzir custos e centrar-se em competências essenciais.

Pereira, Scur \& Mello (2017), considera também que, as vantagens de utilizar o outsourcing são: concentrar os recursos e capacidades nas tarefas essenciais, de forma a poder contribuir com um valor único e superior, aos clientes. Desta forma, a empresa protege e fortalece as suas competências essenciais e vantagem competitiva sustentável, permite o acesso a recursos ou capacidades, que de outra forma não estariam disponíveis, ou não seriam facilmente desenvolvidos internamente.

\section{METODOLOGIA}

Este estudo constitui uma revisão bibliográfica de caráter analítico a respeito das práticas do Outsourcing, nas atividades de desenvolvimento de novos produtos.

A procura de artigos sobre a temática do Outsourcing foi realizada entre outubro de 2018 e junho de 2019 , utilizando-se para a pesquisa as bases de dados da B-ON (Biblioteca de Conhecimento Online). Foi definido como critério de inclusão, artigos publicados entre os anos de 2003 e 2018. Esta escolha foi definida pelos autores.

Os artigos selecionados, tinham que conter conceitos, como: Outsourcing, Inovação, Desenvolvimento de novos produtos, Business Process Outsourcing e combinações entre ambos.

Para a pesquisa na base de dados, B-ON, foi identificado que as publicações em inglês, eram as que mais continham informações relevantes ao estudo. No entanto, o idioma não foi limitativo na obtenção da informação.

Após encontrados os artigos na base de dados, estes foram selecionados, de acordo com os critérios de inclusão previamente definidos.

O processo utilizado foi o seguinte: leitura exploratória, leitura seletiva e escolha adequada do material (em função dos objetivos e tema do 
estudo), leitura analítica e análise de textos. O processo foi concluído com a leitura e redação. Posteriormente, constituiu-se o corpo do texto.

\section{ESTUDO EMPÍRICO}

A Volkswagem tem a sua sede na Alemanha em Wolfsbrug. O veículo é divido em quatro partes: motor, transmissão, plataforma e carroçaria. Procura-se nas três primeiras uma padronização para todas as marcas e modelos da Volkswagen, com vista a economia de escalas em desenvolvimento e produção. Relativamente às carroçarias, há uma maior liberdade de atuação, já que o carro passa a ser desenhado de acordo com os gostos do consumidor local.

A Alemanha é responsável pelo mercado europeu e por produtos "top" para o mercado Norte Americano. A Volkswagen do Brasil é responsável pela produção e desenvolvimento de automóveis para a América do Sul e Ásia e por veículos motorizados pequenos. O México é responsável pela produção e desenvolvimento de processos de algumas adaptações, e testes para EUA e Canadá. A Volkswagen, atualmente, não tem interesse na instalação de atividades de engenharia na China.

No Brasil, a Volkswagen integra dois departamentos que estão diretamente envolvidos com o desenvolvimento de produtos: planeamento do produto (responsável pelos pré-projetos), desenvolvimento de conceitos e acompanhamento do desenvolvimento de novos produtos e a engenharia do produto.

Tanto o planeamento do produto como a engenharia do produto, estão dependentes do Presidente da Volkswagen do Brasil. Neste, já se realizam atividades de outsourcing pois, ex-funcionários realizam por intermédio das suas empresas de engenharia, o desenvolvimento de produtos. $\mathrm{Na}$ Alemanha estão centralizados os laboratórios de maior investimento ou os mais avançados a nível de tecnologia. Isto deve-se à implementação de laboratórios mais caros e só é viável, perante um grande volume de utilização. Daí que o volume produzido localmente não justifica laboratório exclusivo. Sendo a Alemanha, a sede da Volkswagen, há todo o interesse em manter aí os laboratórios e por conseguinte, os projetos que dele se utilizam sob o seu controlo.

$\mathrm{Na}$ VW do Brasil existem laboratórios que funcionam como um segundo centro de desenvolvimento do grupo: laboratório de calibração, 
rigidez das carroçarias, materiais, emissão de poluentes, motores e crash test.

O Brasil possui ainda um centro de estilo.

O processo de desenvolvimento de novos produtos da VW do Brasil divide-se em cinco fases: a primeira, que envolve a área do planeamento do produto. Nesta fase é desenvolvido o conceito inicial do produto e determinado o mercado alvo e as suas configurações básicas. Depois da definição interna, o conceito é apresentado para a aprovação da matriz.

A segunda fase, validação conceitual, o conceito proposto na fase anterior é avaliado por meio de pré-cálculo de custos e estimativas de investimento e tempo necessários. Nesta fase, para além do departamento de planeamento de produtos, contribuem também, a engenharia de produtos, manufatura, qualidade e departamento de compras.

\section{Figura I}

Estrutura do planeamento e desenvolvimento do produto na Volkswagen do Brasil

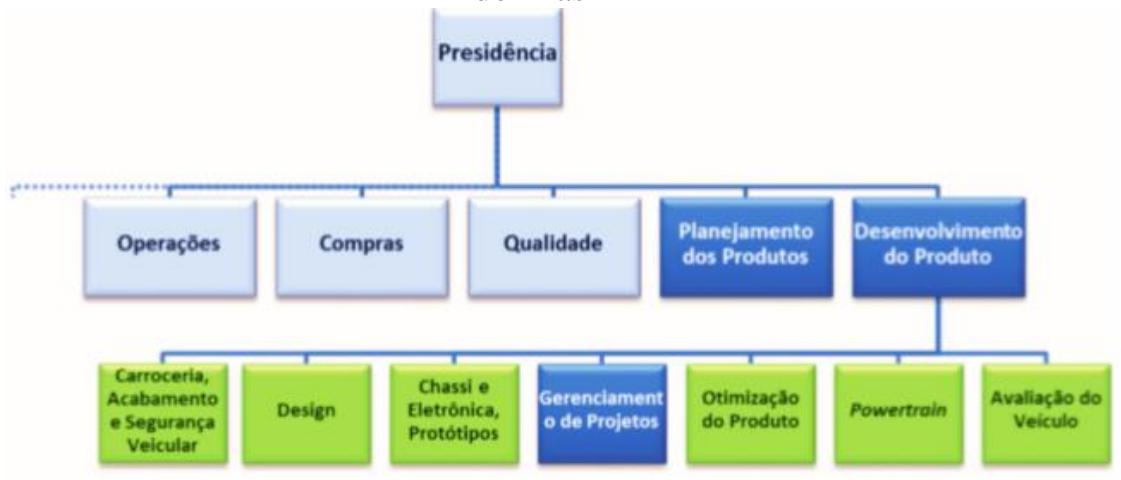

Fonte: Pereira, Scur \& Mello ( 2017)

A terceira fase, aprimoramento, engloba a engenharia do produto. Nesta fase participam as mesmas áreas da fase anterior, sendo a engenharia do produto, a líder.

A quarta fase, implementação da produção, o líder é o Project House, departamento de gestão de projetos, em que os gestores, reportam ao vice presidente de operações. Nesta fase estão presentes as áreas mencionadas 
anteriormente. A última fase, quinta, é a introdução do produto no mercado, este é liderado pelo departamento de marketing.

As razões que levaram à pratica do outsourcing na VW do Brasil foram: falta de capacidade para realizar determinada atividade, carência de recursos, redução de custos e limitação de mão de obra.

Departamentos com atividades mais inovadoras realizam menos outsourcing de atividades, enquanto que outros, que efetuam mais, nomeadamente, as atividades relacionadas com o desempenho de componentes, necessitam de adquirir mão de obra.

O outsourcing na VW do Brasil acontece de duas formas: através da prestação de serviços, no caso de projetos menores e atividades administrativas. Ocorre quando é necessário adquirir mão de obra de engenharia. A outra forma é a empreitada, modalidade utilizada em grandes projetos ou com curto tempo de execução, uma vez que o risco recai sobre o fornecedor.

Este tipo de contratação foi utilizado no projeto de construção do modelo Polo, Polo sedam, Fox e Familia Gold. A subcontratação para desenvolver um veículo completo ainda não aconteceu.

\section{DISCUSSÃO DE RESULTADOS}

O outsourcing pode tornar-se um processo burocrático levando à lentidão, podendo provocar atrasos e impactos nos projetos. Há situações em que o gestor de produção é suficiente para decidir, há outros casos, a maioria, em que a decisão chega até a presidência da empresa. Quando a empresa carece de um determinado recurso e apresenta restrição orçamental dos projetos, tem necessidade de praticar o outsourcing, apesar desta ter alguma competência chave. Sendo a mão de obra interna mais cara do que a contratada, os gestores veem-se "obrigados" a praticar outsourcing para determinadas atividades, uma vez que, a mão de obra contratada tem custos menores. As empresas centram-se, assim, nos contratos de mão de obra para o desenvolvimento de vários programas com diferentes graus de complexidade, flexibilizando o custo de engenharia. As empresas utilizam a estratégia de outsourcing, principalmente quando uma atividade necessita de um conhecimento 
tecnológico que não dispõe, ou não possui recurso internamente (Pereira et al., 2017).

Segundo Mcivor (2011), as empresas só deveram realizar outsourcing se não tiverem experiência ou especialização, para o desenvolvimento de um determinado projeto. Caso contrário, não o deverão fazer. Pois, ao alargar o âmbito das suas atividades, não só prejudica a força da sua competência, como o aumento dos custos da realização da atividade. Weibendaun (2005), também é da opinião que, o outsourcing só deverá ser realizado em atividades secundárias, para as quais a empresa não tem recursos ou experiência, para o desenvolvimento das mesmas.

\section{CONCLUSÃO}

O outsourcing só deve ser realizado para atividades onde a empresa se avalia com capacidade inferior (tradicionalmente aquelas que a análise SWOT deteta como fraquezas organizacionais), isto é, em atividades em que não existe vantagem competitiva. Constitui-se como um meio de inovação no mundo empresarial devendo permitir uma maior eficiência e redução de custos (Mcivor, 2011).

A utilização de BPO é um instrumento de estratégico, que para ser bem sucedido, exige que se conheça, não apenas as necessidades da empresa, mas também, as principais capacidades, que são os critérios essenciais para a seleção de fornecedores (Outsourcing Business Processes for Innovation., 2013).

Com o BPO, ocorre uma melhoria dos serviços, no que respeita ao tempo, ao custo, à qualidade e ao funcionalidade. Os fornecedores que esperam esta melhoria e inovação, utilizam potenciais ferramentas para alcançar mudanças e melhorias significativas (idem).

Procurámos retratar a importância do outsourcing, as suas vantagens e desvantagens e em que situação ou situações deve ser praticado especificando o que nível hierárquico da empresa que deverá tomar a decisão de contratação.

Um dos obstáculos que as organizações enfrentam na seleção de fornecedores, num mercado de fornecedores não desenvolvidos, é a disponibilidade de dados relativas às capacidades dos mesmos. Este constrangimento deve conduzir, de acordo com Aamer, 2018, a uma 
abordagem mais rigorosa na avaliação e seleção dos fornecedores em mercados não desenvolvidos.

O outsourcing deve ser integrado na estratégia empresarial para permitir obter vantagem competitiva, redução de custos e aumento da eficiência. De acordo com Weibendaun, 2005, só deverá ser realizado nas atividades em que a empresa não tem experiência acumulada, nem recursos especializados, sendo o alvo principal desta ação as atividades secundárias. Contudo, pode haver atividades principais nas quais seja importante, em determinado contexto, realizar BPO para atrair/conhecer as melhores práticas do mercado (Marion, Eddleston, Friar \& Deeds, 2015).

A utilização do outsourcing permite ainda concentrar os recursos e capacidades internas nas tarefas essenciais. Desta forma, a empresa protege e fortalece as suas competências essenciais e vantagem competitiva sustentável. Uma limitação deste trabalho consiste na impossibilidade de ter acesso aos dados que estiveram na origem da investigação realizada por Pereira, Scur \& Mello, (2017).

\section{Futuras Linhas de Investigação}

Estudos futuros poderão ser aplicados a outras indústrias de produção do setor automóvel, de outras marcas e em outros países. Deste modo, tornar-se-ia possível comparar os resultados obtidos com o estudo anterior, e assim, poder generalizar os resultados.

\section{BIBLIOGRAFIA}

Acosta, B., Acosta, M., \& Espinoza, B. (2016). Understanding innovation based on company optics: interpretation mistakes on the types of innovation developed. RAI Revista de Administração e Inovação, 13(4), 295-304. https://doi.org/10.1016/j.rai.2016.03.006

Aamer, A. M. (2018). Outsourcing in non-developed supplier markets: a lean thinking approach. International Journal of Production Research, 56(18), 6048-6065. https://doi.org/10.1080/00207543.2018.1465609

Arenhardt, D. L., Simonetto, E. D. O., \& Rodrigues, G. O. (2018). Importance of Innovation for European SMEs: Perception of Experts. Dimensión Empresarial, 16(2), 21-37. https://doi.org/10.15665/dem.v16i2.1450 
Ashok, M., Narula, R., \& Martinez-Noya, A. (2016). How do collaboration and investments in knowledge management affect process innovation in services? Journal of Knowledge Management. https://doi.org/10.1108/JKM-11-2015-0429

Carr, N. G. (2003). TI já não importa. Harvard Business Review Brasil, 9.

Di Gregorio, D., Musteen, M., \& Thomas, D. E. (2009). Offshore outsourcing as a source of international competitiveness for SMEs. Journal of International Business Studies. https://doi.org/10.1057/jibs.2008.90

Feeny, D., Lacity, M., \& Willcocks, L. P. (2005). Taking the Measure of Outsourcing Providers. MIT Sloan Management Review, 46(3), 41-48. Retrieved from http://search.ebscohost.com/login.aspx?direct=true \&db=buh\&AN=1 $7387750 \&$ site $=$ ehost-live

Marion, T. J., Eddleston, K. A., Friar, J. H., \& Deeds, D. (2015). The evolution of interorganizational relationships in emerging ventures: An ethnographic study within the new product development process. Journal of Business Venturing, 30(1), 167-184. https://doi.org/10.1016/j.jbusvent.2014.07.003

Mcivor, R. T. (2011). Considerations on the outsourcing decision: the case of a manufacturing company. To cite this version : $r$ P Fo $r$ R w On ly.

Outsourcing Business Processes for Innovation. (2013). MIT Sloan Management Review, 54(3), 63-69. Retrieved from http://libweb.ben.edu/login?url=http://search.ebscohost.com/login.as px?direct $=$ true $\& d b=$ bth $\& A N=86414900 \&$ site $=$ ehost-live

Oliveira, J. M., Rasia, I. C. B., Silva, D. C. P., \& Patias, T. Z. (2017). Análise das Práticas de Inovação na Área da Saúde: Um Estudo de Caso em Uma Empresa do APL da Saúde na Cidade de Pelotas-RS. Revista de Gestão Em Sistemas de Saúde, 6(1), 52-63. https://doi.org/10.5585/rgss.v6i1.275

Pereira, R. A., Scur, G., \& Mello, F. C. (2017). Outsourcing nas atividades de desenvolvimento de novos produtos: o caso da Volkswagen do Brasil. Exacta, 15(2), 287-301. https://doi.org/10.5585/exactaep.v15n2.6872

Pedriali, M. (2004). Business Process Outsourcing: uma importante ferramenta estratégica da terceirização. In Anais do Congresso Brasileiro de Custos-ABC. 
Silva, M. A. da. (2009). Outsourcing de TI e redefinição do papel da subsidiária: um estudo comparativo entre as subsidiárias brasileira e indiana de uma multinacional americana. JISTEM Journal of Information Systems and Technology Management, 6(2), 173-202. https://doi.org/10.4301/s1807-1775200900020000

Walter, C., Leite, R., \& Craveiro, L. (2015). EQUILÍBRIO, ESTRATÉGIA COMPETITIVA E INOVAÇÃO: UM ESTUDO DESCRITIVO DE CASOS. Gestão e Desenvolvimento, 23 (2015), 49-66

Weidenbaum, M. (2005). Outsourcing: Pros and cons. Business Horizons, 48(4), 311-315. https://doi.org/10.1016/j.bushor.2004.11.001 